\title{
TTM Observations of X1755-338
}

\author{
H. C. Pan $^{1,3}$, G. K. Skinner ${ }^{1}$, R. A. Sunyaev ${ }^{2}$, K. N. Borozdin ${ }^{2}$
}

1 School of Physics and Space Research, University of Birmingham, Edgbaston, Birmingham B15 2TT, UK

2 IKI, Space Research Institute, Profsoyuznaya, 84/32, Moscow 117296, Russia

3 Department of Physics (Theoretical Physics), University of Oxford, 1 Keble Road, Oxford OX1 3NP, UK

\section{Observations}

$\mathrm{X} 1755-338$ is an X-ray binary source which displays $\mathrm{X}$-ray dips with a 4.4 hour period (White et al. 1984). It was previously noted as an unusually soft X-ray source by Jones (1977) and was suggested later as a black-hole candidate (BHC) by White \& Marshall (1984), and White et al. (1984), based on the similarity of its location in an X-ray colour-colour diagram to that of a group of BHCs.

The $T T M$ is a coded-mask imaging spectrometer on board the KVANT module of the MIR space station. It is capable of producing images in the $2-30$ $\mathrm{keV}$ band with an energy resolution of about $18 \%$ at $6 \mathrm{keV}$. The instrumental details are given in Brinkman et al. (1985).

We observed X1755-338 in 1989 March-September during the period of the TTM Galactic Centre Survey.

\section{Results}

As shown in Fig. 1, the source intensity was variable during the observations. Variation of over $30 \%$ was seen on time scales from a few hours to several days. The X-ray intensity in 1989 August-September period appeared to be nearly twice that observed in 1989 March-April. The large data gaps in the TTM light curve prevent us from identifying any $\mathrm{X}$-ray dips.

We have studied the X-ray spectra of X1755-338 accumulated during each observational day. We find that the source spectrum consists of an ultrasoft thermal component and a hard tail above $\sim 6-10 \mathrm{keV}$, which is similar to the high state spectrum of the well known BHCs LMC X-1, LMC X-3 and GX 3394, also observed with the TTM. To our knowledge, this is the first time that a hard tail has been observed from X1755-338.

We have modelled the spectrum with a multi-temperature disc blackbody (Mitsuda et al. 1984) and a power-law. We plot in Fig. 2 the spectrum obtained on 1989 March 31. A significant hard tail is seen above $10 \mathrm{keV}$. We have found that the soft and hard $X$-ray components of X1755-338 vary independently, as in the spectra of LMC X-1, LMC X-3 and GX 339-4 in their high (intensity) state. 
The TTM observations strongly suggest that X1755-338 does indeed belong to the family of BHCs.

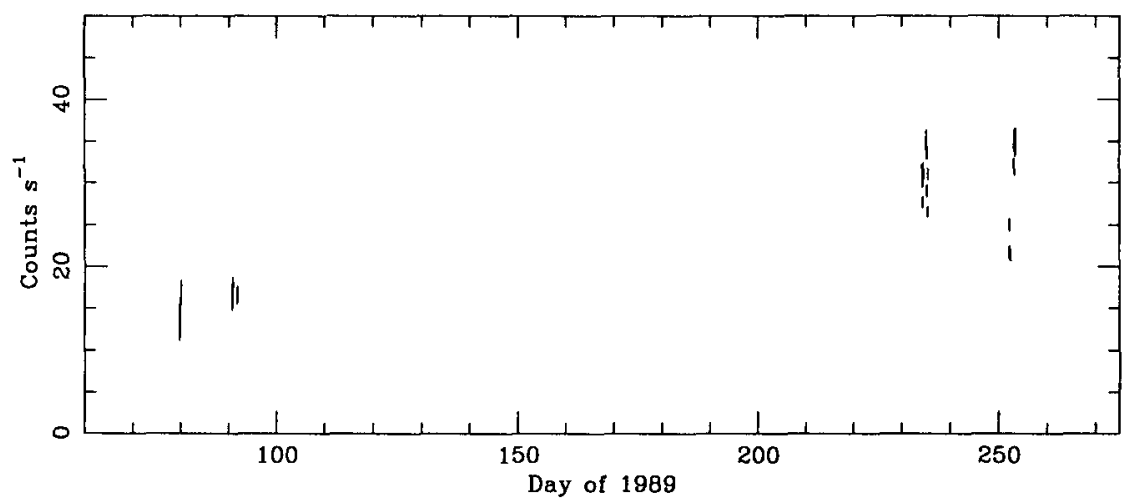

Fig. 1. X-ray $(2-30 \mathrm{keV})$ light curve of X1755-338 in 1989 March-September.

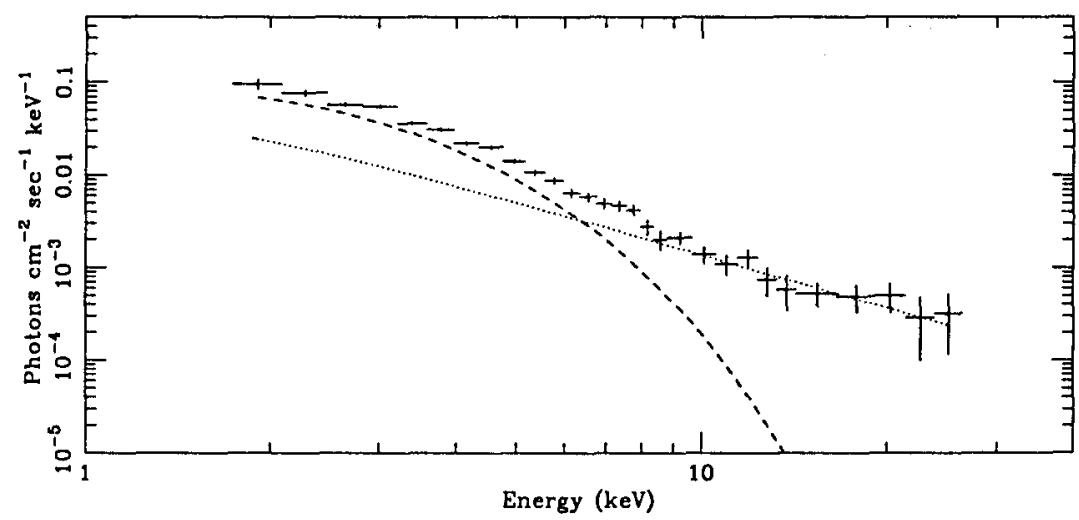

Fig. 2. X-ray spectrum X1755-338 on 1989 March 31 . The soft and hard X-ray components are shown by the dashed line and by the dotted line, respectively.

\section{References}

Brinkman A.C., Dam J., Mels W.A., Skinner G.K., Willmore P.W., 1985, in Nonthermal and Very High Temperature Phenomena in X-Ray Astronomy, Perola G.C. \& Salvati M. (eds), Università "La Sapienza", Roma, p. 263

Jones C., 1977, ApJ 214, 856

Mitsuda K. et al. 1984, PASJ 36, 741

White N. E., Marshall F.E., 1984, ApJ 281, 354

White N.E. et al. 1984, ApJ 283, L9 